\title{
Projetos sociais de empresas no Brasil: arcabouço conceitual para pesquisas empíricas e análises gerenciais*
}

\author{
Renata Buarque Goulart Coutinho** \\ Teresia Diana L. v. A. de Macedo-Soares*** \\ José Roberto Gomes da Silva****
}

S U MÁRIO: 1. Introdução; 2. Metodologia; 3. Referencial teórico; 4. Resultados e discussão; 5 . Considerações finais.

SUMMARY: 1. Introduction; 2. Methodology; 3. Theoretical framework; 4. Results and discussion; 5. Final remarks.

PAlAVRAS-CHAVE: responsabilidade social corporativa; investimentos sociais de empresas; gestão de projetos sociais.

KEY WORDS: corporate social responsibility; corporate social investments; social project management.

Este artigo apresenta o arcabouço conceitual desenvolvido como parte de uma pesquisa com vistas à investigação dos modelos de gestão adotados por empresas no Brasil, na condução de seus projetos sociais, sua adequação às características dos projetos e as implicações para sua efetividade e sustenta-

\footnotetext{
* Artigo recebido em mar. e aceito em ago. 2006.

** Doutora em administração de empresas pelo Instituto de Administração e Gerência, IAG/PUCRio, e administradora do Banco Nacional de Desenvolvimento Econômico e Social (BNDES). Endereço: Av. República do Chile, 100/8 andar — Centro - CEP 20031-917, Rio de Janeiro, RJ, Brasil. E-mail: rbuarque@bndes.gov.br.

*** PhD em filosofia pela Universidade de Montreal, Canadá e professora associada do Instituto de Administração e Gerência, IAG/PUC-Rio. Endereço: Rua Marquês de São Vicente, 225 Gávea — CEP 22453-900, Rio de Janeiro, RJ, Brasil. E-mail: redes@strategy-research.com.

**** Doutor em administração de empresas pelo Instituto de Administração e Gerência, IAG/ PUC-Rio, e professor assistente do IAG/PUC-Rio. Endereço: Rua Marquês de São Vicente, 225 — Gávea — CEP 22453-900, Rio de Janeiro, RJ, Brasil. E-mail: jrgomes@iag.puc-rio.br.
} 
bilidade. Foi feita uma extensa revisão da literatura, a fim de identificar conceitos e construtos que pudessem apoiar a construção do arcabouço. Foi identificada também a interação de diversos fatores que podem trazer implicações para a efetividade dos projetos e para a sustentabilidade. A partir das recomendações de Kerlinger (1973) para operacionalização de conceitos, foram definidos os principais construtos adotados na pesquisa, posteriormente desmembrados em dimensões, às quais, por sua vez, foram atribuídos indicadores. E, por fim, foi visto como o arcabouço resultante pode ser utilizado na condução de pesquisas empíricas sobre projetos sociais desenvolvidos por empresas no Brasil e também sua importância para os gestores de projetos, na análise da adequação entre os diversos fatores intervenientes, com vistas a uma maior efetividade e sustentabilidade dos resultados.

\section{Corporate social projects in Brazil: conceptual framework for empirical research and management analyses}

This article presents a conceptual framework which was developed as part of a research aimed at investigating management models adopted by firms in Brazil to conduct their social projects, how they fit project characteristics, and the implications for their effectiveness and sustainability. Firstly, the article shares the results of a wide-range review of the literature, undertaken to identify concepts and constructs which could support the building of the framework. The research also identifies the interaction of various factors that may have implications for project effectiveness and sustainability. Based on Kerlinger's (1973) recommendations regarding concept operationalization, a description is made of how the main constructs adopted in the research were defined. These were then broken down into dimensions, to which indicators were attributed. An explanation is given of how the resulting framework can be used to guide empirical research into social projects developed by firms in Brazil, and also its importance for project managers in their analyses of the fit between various intervening factors, in order to ensure the greater effectiveness and sustainability of results.

\section{Introdução}

No Brasil, a responsabilidade social corporativa (RSC) foi trazida à tona na década de 1960. A partir dos anos 1980, a RSC voltou às pautas de discussão nos meios empresarial, acadêmico e na mídia. A criação do Grupo de Institutos, Fundações e Empresas (Gife), em 1989; a campanha do Instituto Brasileiro de Análises Sociais e Econômicas (Ibase) pela publicação do balanço social, a partir de 1997; e a criação do Instituto Ethos de Responsabilidade Social, em 1998, foram marcos importantes. No bojo desse processo, está a atuação das 
universidades e institutos de pesquisa. Muitos já contam com centros e grupos dedicados ao estudo da RSC, da ética empresarial e da atuação do terceiro setor.

Apesar do conceito de RSC abranger as relações da organização com seus diversos stakeholders, é seu braço social que mais dá visibilidade às empresas. Muitas são intituladas "empresas socialmente responsáveis" simplesmente por patrocinarem projetos em comunidades. Pouco se questiona sobre a forma como tais projetos são implementados.

Pesquisas mostram que, no Brasil, tem sido crescente a injeção de recursos na área social (Ipea, 2001, 2002; Gife, 2001) e grande parte dos investimentos sociais das empresas é apoiada pelo trabalho voluntário dos empregados (CeatsUSP, 1999). Uma tendência na atuação social das empresas brasileiras é o estabelecimento de parcerias e alianças interinstitucionais e intersetoriais, envolvendo outras empresas, organizações não-governamentais e órgãos do governo (Ceats-USP, 2005). No entanto, ainda predominam atividades de assistência social e de alimentação (Ipea, 2002); nos projetos desenvolvidos por meio de parcerias intersetoriais raramente é feito um planejamento prévio das ações (Fischer et al., 2003) e grande parte das empresas não dispõe de informações sistematizadas sobre o impacto de suas ações sociais (Ipea, 2001). A atuação social das empresas acontece de diversas formas: concentrando-se em um só projeto ou em diversos; por meio de doações esporádicas ou iniciativas de longa duração; e com formas diversas de gestão dos projetos (Fischer et al., 2003; Thompson e co-autores, 2000).

Apesar do crescente número de pesquisas sobre o tema no Brasil, na literatura de RSC, sob a ótica da administração de empresas, ainda é pequena a atenção dispensada à gestão dos projetos sociais, com vistas à obtenção de maior efetividade e sustentabilidade dos resultados.

Na segunda seção, é apresentada a metodologia utilizada para a construção do arcabouço conceitual. Na terceira, são comentadas as principais proposições teóricas que o embasaram e na quarta são apresentados e discutidos os resultados - o arcabouço em si, seus construtos, dimensões e indicadores - e suas possíveis utilizações nos meios acadêmico e empresarial. $\mathrm{Na}$ quinta seção, são tecidas as considerações finais.

\section{Metodologia}

Como já dito, foi feita uma extensa revisão da literatura. Devido à característica interdisciplinar da pesquisa, foi necessário investigar diferentes áreas de conhecimento. Para tanto, foram pesquisados os temas: RSC, ética, desenvolvi- 
mento sustentável, participação social e gestão de projetos sociais. Este artigo, no entanto, limita-se aos temas referentes a desenvolvimento e sustentabilidade e a projetos sociais, por serem mais diretamente relacionados ao arcabouço.

Na literatura das áreas de políticas públicas e desenvolvimento, mais especificamente, foram investigados conceitos e definições relacionados a desenvolvimento sustentável, participação social e gestão de projetos sociais. Inicialmente, foi efetuada uma pesquisa em bases de dados nacionais e internacionais (Search Bank, Ebsco Host, Emerald, ProQuest, Sociological Abstracts, banco de teses da Capes), além de publicações de agências multilaterais de fomento (Cepal, BID, Banco Mundial). Foram investigados também os seguintes periódicos: Revista de Administração Pública, Revista de Administração Municipal, Cadernos de Estudos Sociais, Espaço e Debates e Revista do Serviço Público.

Apoiando-se nas recomendações de Kerlinger (1973) para operacionalização de conceitos, foram definidos, com base na revisão da literatura, os principais construtos adotados na pesquisa, posteriormente desmembrados em dimensões, às quais, por sua vez, foram atribuídos indicadores.

\section{Referencial teórico}

\section{Desenvolvimento e sustentabilidade}

O conceito de desenvolvimento sustentável traz em seu bojo contradições que refletem a tensão entre crescimento e limite (Ismerio, 1999). A noção de desenvolvimento remete ao aumento, crescimento. Por sua vez, a noção de sustentabilidade tem em si a idéia de limites, tanto externos - exaustão de recursos, poluição - , quanto internos - inerentes as sociedades humanas e seu universo simbólico, não-material, cultural. Apesar do desenvolvimento remeter à idéia de crescimento, implica mudanças qualitativas do sistema social (Tankersley, 1994). Do ponto de vista social, o mesmo ritmo de crescimento pode culminar no desenvolvimento, no "maldesenvolvimento" ou no "de-desenvolvimento" (Sachs, 2000).

A noção de desenvolvimento sustentável integra as propostas do ecodesenvolvimento e do desenvolvimento endógeno e local, abarcando as dimensões econômica, política, tecnológica, ecológica e cultural, constitutivas de toda a sociedade humana. Envolve, portanto, objetivos situados no tripé eqüidade social-conservação ambiental-eficiência econômica. 
O conceito de desenvolvimento sustentável, apesar de amplamente discutido, ainda é de difícil operacionalização. A análise da sustentabilidade de uma iniciativa, programa ou ação social é tarefa árdua, em função da dificuldade de se encontrarem indicadores que possam ser utilizados para tal mensuração. A sustentabilidade pode ser vista de forma macro, analisando-se a estratégia generalizada de consumo, crescimento e desenvolvimento da sociedade, ou de maneira micro, analisando-se um determinado processo, programa ou projeto (Mokate, 2002). Este último é o enfoque adotado neste artigo.

A visão micro da sustentabilidade tem sido muito utilizada pelas organizações multilaterais de financiamento a programas e projetos de desenvolvimento. O Banco Mundial, a partir de 1990, propôs uma interpretação de sustentabilidade que faz referência a seis tipos de capital: humano, natural, cultural, institucional, físico e financeiro. A sustentabilidade de um programa ou projeto dependeria, portanto, de um equilíbrio apropriado entre os diversos tipos de capital. Tal interpretação faz uma ponte com as definições mais macro da sustentabilidade, ao reconhecer uma relação de mão-dupla entre o entorno e a iniciativa que se analisa, já que invoca a necessidade de harmonia entre ambos (Mokate, 2002).

Em consonância com essa interpretação, o Banco Mundial passou, então, a qualificar a sustentabilidade de seus programas e projetos, segundo um amplo conjunto de fatores que são analisados (Mokate, 2002): elementos externos; fatores econômicos; financeiros; técnicos; sociais; ambientais; institucionais; e de governabilidade.

\section{Projetos sociais}

\section{Conceitos e classificações}

Um projeto social busca, por meio de um conjunto integrado de atividades, transformar uma parcela da realidade, reduzindo ou eliminando um déficit, ou solucionando um problema, para satisfazer necessidades de grupos que não possuem meios para solucioná-las por intermédio do mercado (Cepal, 1995; Nogueira, 1998).

Freqüentemente, os termos projetos, programas e políticas sociais são confundidos. Um programa social é um conjunto de projetos; e uma política social, por sua vez, é um conjunto de programas. Projetos e programas são a tradução operacional das políticas sociais. Um projeto envolve ações concretas a serem desenvolvidas em um horizonte de tempo e espaço determina- 
dos, restritas pelos recursos disponíveis para tal. Os programas, em geral, envolvem horizontes de tempo mais longos que os projetos. Pode-se, portanto, analisar um programa por meio do estudo dos projetos que o compõem (Cepal, 1995, 1998).

Faz-se necessária, também, uma distinção entre as áreas de atuação dos projetos, pois esse universo é muito vasto e heterogêneo. Embora não haja consenso sobre a questão, foram pesquisadas algumas fontes - documentos do Prêmio ECO-Amcham, Gife (2001), Ceats-USP (1999, 2005), Ipea (2002) e o Guia Exame de Boa Cidadania Corporativa, com o intuito de se chegar a um denominador comum. Essas fontes tratam de projetos sociais relacionados à iniciativa privada, no contexto brasileiro, o que permitiu uma melhor adequação da terminologia ao campo deste artigo. As duas maneiras mais usuais de se classificar os projetos são: com relação ao seu objeto principal (saúde, educação, meio ambiente, cultura) ou com relação às características distintivas da população-alvo (crianças, adolescentes, portadores de deficiência, idosos).

Com relação às etapas do ciclo de um projeto, muitas são as divisões apresentadas pelos autores investigados. Este artigo adota a seguinte divisão, sugerida pelo Banco Interamericano de Desenvolvimento (BID): identificação de idéias do projeto, definição de objetivos, desenho, análise e aprovação, execução e avaliação ex post (BID/Ilpes, 2000).

\section{Critérios de avaliação}

Atenção especial deve ser dada ao processo de avaliação, que permite alcançar de forma mais adequada os resultados, com melhor utilização dos recursos, além de munir os formuladores e gestores de informações importantes para o desenho de futuras iniciativas ou para correção de cursos de atuação, como um mecanismo de retroalimentação (Cohen e Franco, 1998).

São inúmeras as sugestões a respeito dos critérios para a avaliação de projetos sociais. Alguns, no entanto, permanecem definidos de forma difusa e de difícil operacionalização, além de existirem muitas divergências em torno de suas definições.

O critério mais comumente utilizado é a eficácia (Mokate, 2002). É também um dos únicos acerca do qual há consenso. Refere-se ao grau em que se atingem os objetivos de um projeto em um período de tempo, com a qualidade esperada, independentemente de seus custos (Cepal, 1998; Cohen e Franco, 1998; Mokate, 2002).

O conceito de eficiência envolve a relação entre duas dimensões básicas: resultados do projeto (bens e serviços produzidos) e recursos utilizados (insumos e atividades). Refere-se à maneira como os objetivos são alcançados e remete à capacidade de selecionar e usar os melhores meios, com os 
menores custos possíveis, para se realizar uma tarefa ou propósito (Cepal, 1998; Cohen e Franco, 1998; Mokate, 2002).

Vale ressaltar que, no caso de projetos sociais e ambientais, um custo pode ser incorrido pelo desgaste ou sacrifício de um recurso, seja ele tangível ou intangível: tempo, recurso ambiental, recurso financeiro, capital social, confiança (Mokate, 2002). Nesse caso, custos e benefícios não deveriam ser medidos apenas em termos financeiros, devendo também ser considerados segundo dimensões sociais e psicológicas (Narayan, 1995).

Com relação à efetividade, cada autor estudado mostra uma interpretação diferente. Para Mokate (2002), eficácia e efetividade são sinônimas. Para a Unicef, efetividade e impacto podem ser considerados sinônimos, e expressam quanto o projeto tem efeitos (positivos) no ambiente externo (Costa e Castanhar, 2003). Cohen e Franco (1998) sugerem que a efetividade é a relação entre os resultados e o objetivo. A Cepal (1998:18) considera que a efetividade supõe a eficiência e a eficácia e a define como "a capacidade organizacional para ser eficiente e eficaz ao longo do tempo, alcançando níveis de impacto elevados e sustentados". Neste artigo, efetividade é considerada uma medida geral de desempenho do projeto, desmembrada em eficácia e eficiência.

\section{Gestão de projetos sociais}

A gestão do projeto consiste em dar unidade e coerência ao ciclo de ação. Um projeto tem como conseqüência um conjunto de produtos (gerados via processos de conversão), resultados (previstos nos objetivos e metas) e impactos (mudanças na realidade efetivamente alcançadas). A gestão deve assegurar que tais produtos, resultados e impactos sejam coerentes com a concepção e os fins do projeto, garantindo sua eficácia e efetividade, por meio de uma adequada combinação de recursos (Nogueira, 1998).

Cabe considerar que: os processos de conversão são implementados em uma estrutura social e material específica; a interação dos membros da estrutura social gera cultura e clima organizacional também específicos; junto com os processos, os atores da estrutura cumprem uma série de papéis funcionais que permitem articular as ações para maximizar o atingimento dos objetivos; o programa está inserido em um contexto com o qual interage em maior ou menor grau; a interação de todos os elementos descritos gera um modelo específico de organização e gestão em cada programa social (Cepal, 1998). 

mensões:

Nogueira (1998) propõe uma tipologia de projetos baseada em duas di-

v a capacidade de programação das tarefas — dada pelas exigências de variação em sua execução, diferindo com relação ao grau em que são suscetíveis à rotinização ou formalização;

v a interação com os destinatários da ação — determina a relação entre operador e destinatário, assim como o nível de mudança que se pretende provocar nas condições ou capacidades dos destinatários.

Pode-se imaginar que quanto maior for o âmbito de comportamentos que se deseja impactar, das atitudes a serem modificadas e dos valores a serem estabelecidos, maior será a necessidade de interação entre a populaçãoobjetivo e os operadores do projeto, com maior discricionariedade no plano da gestão, gerando uma maior necessidade da criação de mecanismos para participação da população (Nogueira, 1998).

A Cepal (1998) propõe uma tipologia de modelos de organização e gestão de projetos sociais que considera duas dimensões:

v o grau de padronização dos produtos (bens ou serviços resultantes do projeto);

v a homogeneidade/heterogeneidade da população-objetivo - nível de semelhança nas "variáveis pertinentes" que afetam os objetivos de impacto do programa.

É possível, portanto, fazer uma associação entre as duas tipologias citadas - de projetos e de modelos de organização e gestão.

\section{Resultados e discussão}

\section{Arcabouço conceitual}

A figura representa o arcabouço conceitual construído para este artigo, ilustrando de maneira simplificada a interação de diversos fatores que podem trazer implicações para a efetividade dos projetos sociais das empresas no Brasil e para a sustentabilidade. 
Arcabouço conceitual

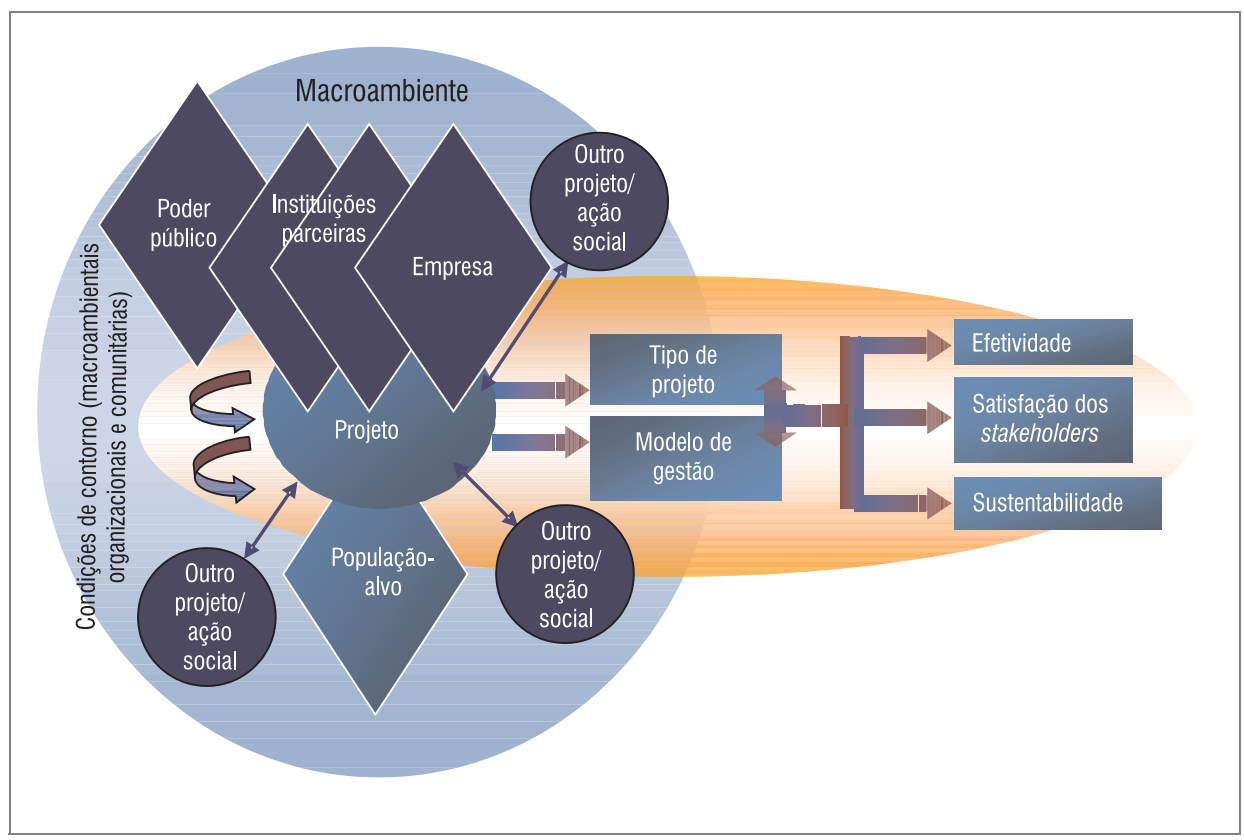

Embora reconheça a complexidade de tais interações, este artigo concentra-se nos fatores situados na região alaranjada da figura, ou seja, nos modelos de gestão adotados, na sua adequação às características dos projetos e nas conseqüências para a efetividade e sua contribuição para a sustentabilidade. Também abrange, de forma menos intensa, os outros fatores, já que alguns podem ser considerados elementos constitutivos dos próprios projetos, pois projetos só existem porque também há populações situadas em um contexto histórico, cultural, ambiental e socioeconômico específico, com necessidades a serem satisfeitas. Os projetos não se situam nesse contexto de forma isolada. Provavelmente existirão outras ações e instituições que tencionam atacar os mesmos problemas, de forma direta ou indireta, articuladas ou não com o projeto em questão. Ademais, são de fundamental relevância a atuação do poder público e a forma de interação do projeto com suas diversas esferas.

Portanto, o projeto, representado pelo círculo central na figura, é resultante de uma rede de relacionamentos entre a principal empresa fomentadora, as empresas e instituições parceiras no projeto (organizações sem fins lucrativos, órgãos e instâncias governamentais), a comunidade ou comunidades envolvidas ou beneficiadas e outros projetos que tenham ações complementares às suas. Todas essas relações acontecem em um contexto mais amplo — o macroambiente. 
A partir da revisão da literatura, parte-se do pressuposto que a combinação dos fatores macroambientais, comunitários e organizacionais (relacionados às instituições envolvidas no projeto) influencia a caracterização do projeto - aqui denominada tipo de projeto - e o modelo de gestão adotado, que, por sua vez, também influenciam-se mutuamente. Daí o fato de sua relação ser representada por uma seta de mão-dupla.

O arcabouço também sugere que o tipo de projeto e o modelo de gestão adotado (consideradas as variáveis principais) influenciam de alguma forma a efetividade, a satisfação dos stakeholders e a sustentabilidade dos projetos (variáveis de desempenho), embora não sejam suficientes para explicar o desempenho do projeto em sua totalidade. O desempenho também sofre influência indireta das condições de contorno representadas na figura.

\section{Construtos adotados na pesquisa, dimensões constitutivas e indicadores}

Para que fosse possível aplicar o arcabouço a pesquisas empíricas e a análises gerenciais, foi necessário um detalhamento, especificando-se suas dimensões, construtos e indicadores, como mostra o quadro 1.

Para o conceito de projeto social - conceito central do artigo - adotou-se a seguinte definição, construída a partir das definições apresentadas por Nogueira (1998) e Cepal (1995, 1998):

\footnotetext{
Um projeto social é um conjunto de ações que têm por propósito provocar impactos sobre indivíduos ou grupos denominados população-alvo ou beneficiários, que compreendem uma determinada destinação de recursos e responsabilidades em um período de tempo determinado.
}

Os construtos tipo de projeto e modelo de organização e gestão tomaram como base os trabalhos de Nogueira (1998) e Cepal (1998), com adaptações, em função de sua adequação ao objeto deste artigo. O construto configuração institucional, por sua vez, foi uma construção dos autores e está representado na figura pelo conjunto das formas em preto. Para o construto efetividade, adota-se a sugestão da Cepal (1998), considerarmos que seja a mais abrangente e de mais fácil compreensão. O construto satisfação dos stakeholders foi uma construção dos autores, baseada na literatura sobre teoria dos stakeholders e na gestão estratégica voltada para eles, em que a satisfação dos diversos públicos de interesse é considerada medida-chave de desempenho. 


\section{Quadro 1}

\section{Construtos adotados na pesquisa e suas definições operacionais}

\begin{tabular}{ll}
\hline Construto & Definição operacional \\
\hline Tipo de projeto & $\begin{array}{l}\text { Caracterização do projeto, de acordo com sua área de atuação, características } \\
\text { distintivas e grau de homogeneidade da população-alvo, seu objetivo principal, } \\
\text { nível de impacto que pretende causar, produtos resultantes e grau de } \\
\text { padronização destes produtos, tipo e quantidade de recursos utilizados e o grau } \\
\text { de rotinização e de complexidade das tarefas executadas. } \\
\text { Modelo adotado para gerenciar o projeto, caracterizado pela orientação básica } \\
\text { organização } \\
\text { e gestão } \\
\text { trabalho, estrutura organizacional adotada, conhecimento necessário e } \\
\text { capacitação da equipe do projeto, grau de flexibilidade, forma de liderança e grau } \\
\text { de participação dos beneficiários e da equipe operacional do projeto. } \\
\text { Configuração } \\
\text { institucional } \\
\text { instituiçãa operadora do projeto, instituições parceiras, articulação com o poder } \\
\text { público e outros projetos ou ações sociais que tenham ações complementares ao } \\
\text { projeto. } \\
\text { Medida de desempenho do projeto, desmembrada em eficácia (grau em que se } \\
\text { atingem os objetivos do projeto em um período de tempo, com a qualidade } \\
\text { esperada) e eficiência (relação entre os resultados do projeto e os recursos } \\
\text { utilizados). } \\
\text { Capacidade de continuidade do projeto e de seus resultados ao longo do tempo, } \\
\text { stakeholders } \\
\text { influenciada por fatores financeiros, sociais, institucionais e de governabilidade. } \\
\text { Grau de satisfação dos diversos públicos afetados pelo projeto, com relação aos } \\
\text { resultados obtidos: população-alvo, comunidade local, equipe do projeto, } \\
\text { empregados das empresas, instituições parceiras, poder público e outros } \\
\text { públicos considerados relevantes. }\end{array}$ \\
\hline
\end{tabular}

Para o construto sustentabilidade, a partir dos fatores utilizados pelo Banco Mundial para avaliar a sustentabilidade de suas iniciativas de desenvolvimento, foi feito um recorte que inclui apenas fatores que, de alguma forma, sejam influenciados pela combinação entre o tipo de projeto, o modelo de gestão adotado e a configuração institucional do projeto. O intuito aqui não é medir a sustentabilidade do projeto de forma absoluta, mas captar indícios da contribuição dos fatores pesquisados à sustentabilidade.

Nos quadros 2 a 7 são apresentados os construtos utilizados no arcabouço conceitual, desmembrados em suas dimensões constitutivas e indicadores. São apresentados também os valores e categorias que cada indicador pode assumir. A especificação de dimensões para cada construto é uma cons- 
trução dos autores, a partir da literatura investigada, da experiência prática e de estudos anteriores a respeito do tema. No caso dos construtos tipo de projeto e modelo de gestão, as dimensões são, em sua maioria, adaptações das sugestões de Nogueira (1998) e Cepal (1998).

As dimensões relativas ao construto configuração institucional devem ser analisadas de forma qualitativa. Os resultados obtidos devem ser cruzados com os dos demais construtos.

O construto tipo de projeto é composto por dimensões que necessitam ser analisadas qualitativa e quantitativamente. Recomendamos que seja efetuada a análise da adequação entre os construtos tipo de projeto e modelo de gestão, conforme os quadros 8 e 9. De acordo com a literatura investigada, aos tipos 1, 2, 3 e 4 de projetos (quadro 8) devem corresponder os modelos burocrático, clientelar, adhocrático e de integração homogênea, respectivamente (quadro 9). É importante ressaltar que não se deve esperar que tal correspondência seja perfeita, mas que haja algum grau de correlação entre os dois construtos para um mesmo projeto, o que denotaria coerência na gestão. Por fim, os resultados dos construtos efetividade, satisfação dos stakeholders e sustentabilidade devem ser cruzados com os resultados dos demais construtos e com a análise de adequação entre os tipos de projeto e os modelos de gestão detectados.

\begin{tabular}{|c|c|c|}
\hline \multicolumn{3}{|c|}{ Quadro 2} \\
\hline Dimensões & Indicadores & Valores/Categorias \\
\hline \multirow{3}{*}{$\begin{array}{l}\text { Empresa ou empresas } \\
\text { patrocinadoras do projeto }\end{array}$} & Razão social & \\
\hline & Principal setor de atuação & $\begin{array}{ll}\nabla & \text { Indústria } \\
\nabla & \text { Comércio } \\
\nabla & \text { Prestação de serviços } \\
\nabla & \text { Intermediação financeira } \\
\nabla & \text { Outro }\end{array}$ \\
\hline & $\begin{array}{l}\text { Grau de envolvimento da(s) } \\
\text { empresa(s) no projeto }\end{array}$ & 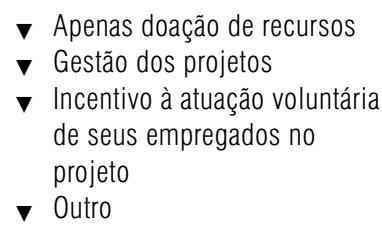 \\
\hline
\end{tabular}




\begin{tabular}{|c|c|c|}
\hline Dimensões & Indicadores & Valores/Categorias \\
\hline \multirow[t]{2}{*}{ Instituição operadora do projeto } & Razão social & \\
\hline & Tipo & 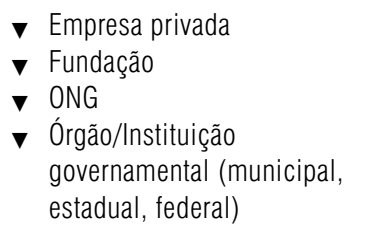 \\
\hline \multirow[t]{2}{*}{ Instituições parceiras } & Razão social & \\
\hline & Tipo & 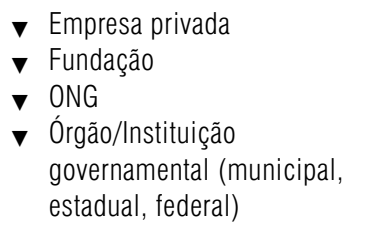 \\
\hline \multirow[t]{2}{*}{ Articulação com o poder público } & Órgão/Instituição governamental & $\begin{array}{ll}\boldsymbol{\nabla} & \text { Municipal } \\
\boldsymbol{\nabla} & \text { Estadual } \\
\boldsymbol{\nabla} & \text { Federal }\end{array}$ \\
\hline & Objetivo da articulação & $\boldsymbol{\nabla}$ Motivo da articulação \\
\hline \multirow[t]{2}{*}{$\begin{array}{l}\text { Articulação com outros projetos/ } \\
\text { ações sociais }\end{array}$} & Descrição dos projetos & 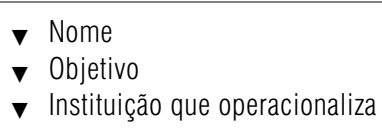 \\
\hline & Objetivos da articulação & $\boldsymbol{\nabla}$ Motivo da articulação \\
\hline
\end{tabular}

\begin{tabular}{|lll|}
\hline \multicolumn{2}{c|}{ Quadro 3} \\
& Tipo de projeto: dimensões e indicadores \\
\hline Dimensões & Indicadores & Valores/Categorias \\
\hline Área de atuação & Objeto de atuação do projeto & $\boldsymbol{\nabla}$ Educação \\
& $\boldsymbol{\nabla}$ Cultura \\
& $\boldsymbol{\nabla}$ Meio ambiente \\
& $\boldsymbol{\nabla}$ Saúde \\
& $\boldsymbol{\nabla}$ Esportes \\
& $\boldsymbol{\nabla}$ Geração de renda \\
& $\boldsymbol{\nabla}$ Assistência social \\
& $\boldsymbol{\nabla}$ Outro
\end{tabular}

Rap Rio de Janeiro 40(5):763-87, Set./Out. 2006 


\begin{tabular}{|c|c|c|}
\hline Dimensões & Indicadores & Valores/Categorias \\
\hline População-alvo & $\begin{array}{l}\text { Características distintivas - } \\
\text { grupo a que pertence } \\
\text { Grau de homogeneidade das } \\
\text { características relevantes } \\
\text { para o projeto }\end{array}$ & $\begin{array}{l}\boldsymbol{\nabla} \text { Crianças } \\
\boldsymbol{\nabla} \text { Adolescentes e/ou jovens } \\
\boldsymbol{\nabla} \text { Adultos } \\
\boldsymbol{\nabla} \text { Famílias } \\
\boldsymbol{\nabla} \text { Terceira idade } \\
\boldsymbol{\nabla} \text { Mulheres } \\
\nabla \text { Minorias étnicas } \\
\boldsymbol{\nabla} \text { Outros } \\
\boldsymbol{\nabla} \text { Homogênea } \\
\boldsymbol{\nabla} \text { Heterogênea }\end{array}$ \\
\hline Objetivo principal & $\begin{array}{l}\text { Descrição do objetivo } \\
\text { Tipo de objetivo }\end{array}$ & 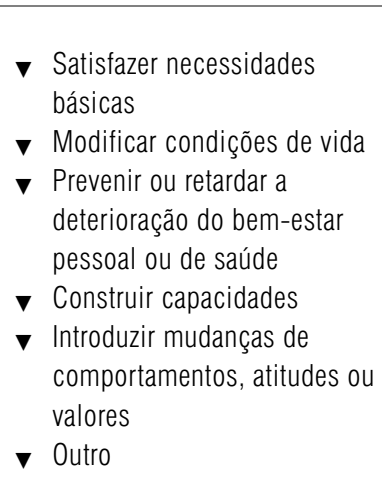 \\
\hline Impacto & $\begin{array}{l}\text { Nível de impacto que pretende } \\
\text { causar }\end{array}$ & $\begin{array}{l}\text { จ Profundo (alterar atributos } \\
\text { pessoais dos beneficiários) } \\
\text { v Superficial (não alterar } \\
\text { atributos pessoais dos } \\
\text { beneficiários) }\end{array}$ \\
\hline Produtos resultantes & $\begin{array}{l}\text { Descrição dos produtos } \\
\text { Grau de padronização dos produtos }\end{array}$ & $\begin{array}{ll}\text { Vadronizados } \\
\text { Vão-padronizados } \\
\text { (customizados) }\end{array}$ \\
\hline Recursos & $\begin{array}{l}\text { Tipo e quantidade de recursos } \\
\text { destinados ao projeto pelas } \\
\text { instituições envolvidas }\end{array}$ & $\begin{array}{ll}\text { v } & \text { Físicos } \\
\text { v } & \text { Financeiros } \\
\text { v } & \text { Humanos } \\
\text { v } & \text { Tecnológicos }\end{array}$ \\
\hline Atividades e tarefas & Grau de complexidade & 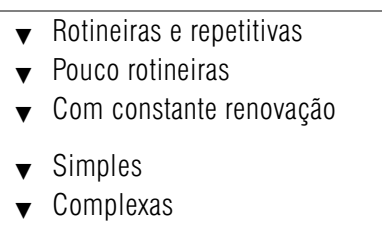 \\
\hline
\end{tabular}

Rap Rio de Janeiro 40(5):763-87, Set./Out. 2006 


\begin{tabular}{|c|c|c|}
\hline \multicolumn{2}{|r|}{ Quadro 4} & Modelo de organização e gestão: dimensões e indicadores \\
\hline Dimensões & Indicadores & Valores/Categorias \\
\hline Orientação da gestão & Para oferta/para demanda & 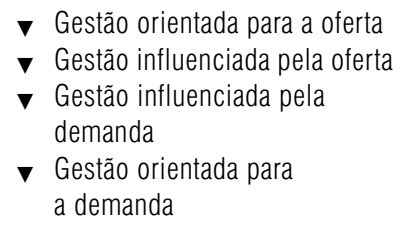 \\
\hline \multirow[t]{3}{*}{$\begin{array}{l}\text { Controle e medição de } \\
\text { desempenho }\end{array}$} & Mecanismos de controle & $\begin{array}{l}\text { Vupervisão direta } \\
\text { e hierárquica } \\
\text { v Auto-supervisão e autocontrole } \\
\text { de grupos de trabalho } \\
\text { v Sistemas de controle e gestão } \\
\text { v Padrões de serviço e satisfação } \\
\text { do beneficiário }\end{array}$ \\
\hline & $\begin{array}{l}\text { Critério básico para medição } \\
\text { de desempenho }\end{array}$ & $\begin{array}{l}\text { V Eficácia e eficiência } \\
\text { v Aprendizagem } \\
\text { v Coordenação e gestão de redes } \\
\text { v Satisfação dos stakeholders }\end{array}$ \\
\hline & Indicadores de resultado & \\
\hline \multirow[t]{2}{*}{ Organização do trabalho } & Estímulo ao trabalho em equipe & $\begin{array}{ll}\boldsymbol{\nabla} & \text { Baixo } \\
\boldsymbol{\nabla} & \text { Moderado } \\
\boldsymbol{\nabla} & \text { Alto }\end{array}$ \\
\hline & Métodos de trabalho & $\begin{array}{ll}\text { v } & \text { Programáveis } \\
\text { v Não-programáveis } \\
\text { v } \text { Mistos }\end{array}$ \\
\hline Estrutura & Departamentalização & $\begin{array}{l}\text { V Funcional } \\
\text { v Equipes de trabalho, matricial } \\
\boldsymbol{\nabla} \text { Divisões territoriais } \\
\boldsymbol{\nabla} \text { Por produto/por cliente }\end{array}$ \\
\hline Equipe do projeto & Conhecimento necessário & 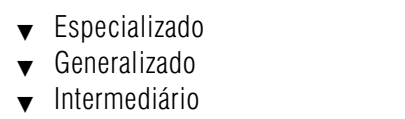 \\
\hline
\end{tabular}

Rap Rio de Janeiro 40(5):763-87, Set./Out. 2006 


\begin{tabular}{|c|c|c|}
\hline Dimensões & Indicadores & Valores/Categorias \\
\hline \multirow[t]{2}{*}{ Equipe do projeto } & $\begin{array}{l}\text { Comprometimento das pessoas } \\
\text { com os objetivos organizacionais }\end{array}$ & $\begin{array}{l}\boldsymbol{\nabla} \text { Baixo } \\
\boldsymbol{\nabla} \text { Moderado } \\
\boldsymbol{\nabla} \text { Alto }\end{array}$ \\
\hline & $\begin{array}{l}\text { Interesse da gestão pela } \\
\text { capacitação das pessoas }\end{array}$ & $\begin{array}{ll}\boldsymbol{\nabla} & \text { Baixo } \\
\boldsymbol{\nabla} & \text { Moderado } \\
\boldsymbol{\nabla} & \text { Alto }\end{array}$ \\
\hline \multirow[t]{2}{*}{$\begin{array}{l}\text { Flexibilidade e percepção } \\
\text { do contexto }\end{array}$} & Propensão a mudanças & $\begin{array}{ll}\boldsymbol{\nabla} & \text { Baixa (estável) } \\
\boldsymbol{\nabla} & \text { Alta (dinâmica) } \\
\boldsymbol{\nabla} & \text { Intermediária }\end{array}$ \\
\hline & Contexto & $\begin{array}{l}\text { V Estável e previsível } \\
\text { จ Estável, mas com } \\
\text { aleatoriedade } \\
\text { จ Turbulento, incerto }\end{array}$ \\
\hline \multirow[t]{3}{*}{ Liderança } & Estilo & 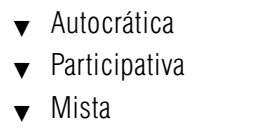 \\
\hline & Processo de decisão & $\begin{array}{ll}\boldsymbol{\nabla} & \text { Centralizado } \\
\boldsymbol{\nabla} & \text { Descentralizado }\end{array}$ \\
\hline & Canal para resolução de conflitos & $\begin{array}{ll} & \text { Hierarquia } \\
\text { v Equipes de trabalho } \\
\text { v Comitê }\end{array}$ \\
\hline \multirow[t]{3}{*}{$\begin{array}{l}\text { Participação dos beneficiários } \\
\text { (população-alvo) }\end{array}$} & $\begin{array}{l}\text { Na geração de idéias para } \\
\text { o projeto }\end{array}$ & $\begin{array}{ll}\boldsymbol{\nabla} & \text { Alta } \\
\boldsymbol{\nabla} & \text { Variável } \\
\boldsymbol{\nabla} & \text { Baixa }\end{array}$ \\
\hline & $\begin{array}{l}\text { Na definição dos objetivos do } \\
\text { projeto }\end{array}$ & $\begin{array}{ll}\boldsymbol{\nabla} & \text { Alta } \\
\boldsymbol{\nabla} & \text { Variável } \\
\boldsymbol{\nabla} & \text { Baixa }\end{array}$ \\
\hline & Na concepção/desenho do projeto & 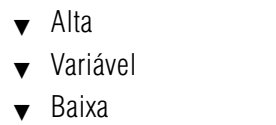 \\
\hline
\end{tabular}

continua

Rap Rio de Janeiro 40(5):763-87, Set./Out. 2006 


\begin{tabular}{|c|c|c|}
\hline Dimensões & Indicadores & Valores/Categorias \\
\hline \multirow[t]{4}{*}{$\begin{array}{l}\text { Participação dos beneficiários } \\
\text { (população-alvo) }\end{array}$} & Na análise e aprovação do projeto & $\begin{array}{ll}\boldsymbol{\nabla} & \text { Alta } \\
\boldsymbol{\nabla} & \text { Variável } \\
\boldsymbol{\nabla} & \text { Baixa }\end{array}$ \\
\hline & $\begin{array}{l}\text { Na execução/implementação } \\
\text { do projeto }\end{array}$ & $\begin{array}{ll}\boldsymbol{\nabla} & \text { Alta } \\
\boldsymbol{\nabla} & \text { Variável } \\
\boldsymbol{\nabla} & \text { Baixa }\end{array}$ \\
\hline & $\begin{array}{l}\mathrm{Na} \text { avaliação dos resultados } \\
\text { do projeto }\end{array}$ & 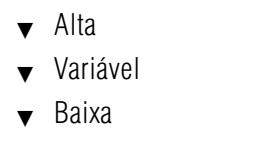 \\
\hline & $\begin{array}{l}\text { Instância máxima de } \\
\text { participação da } \\
\text { população-alvo }\end{array}$ & $\begin{array}{l}\text { V } \text { Compartilhamento } \\
\text { de informações } \\
\text { v Consulta } \\
\text { v Tomada de decisão } \\
\text { v Ação }\end{array}$ \\
\hline \multirow[t]{6}{*}{$\begin{array}{l}\text { Participação da equipe } \\
\text { operacional do projeto }\end{array}$} & $\begin{array}{l}\text { Na geração de idéias para } 0 \\
\text { projeto }\end{array}$ & $\begin{array}{ll}\boldsymbol{\nabla} & \text { Alta } \\
\boldsymbol{\nabla} & \text { Variável } \\
\boldsymbol{\nabla} & \text { Baixa }\end{array}$ \\
\hline & $\begin{array}{l}\text { Na definição dos objetivos } \\
\text { do projeto }\end{array}$ & $\begin{array}{ll}\boldsymbol{\nabla} & \text { Alta } \\
\boldsymbol{\nabla} & \text { Variável } \\
\boldsymbol{\nabla} & \text { Baixa }\end{array}$ \\
\hline & $\begin{array}{l}\text { Na concepção/desenho } \\
\text { do projeto }\end{array}$ & $\begin{array}{ll}\boldsymbol{\nabla} & \text { Alta } \\
\boldsymbol{\nabla} & \text { Variável } \\
\boldsymbol{\nabla} & \text { Baixa }\end{array}$ \\
\hline & $\begin{array}{l}\text { Na análise e aprovação } \\
\text { do projeto }\end{array}$ & 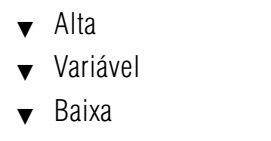 \\
\hline & $\begin{array}{l}\text { Na execução/implementação } \\
\text { do projeto }\end{array}$ & 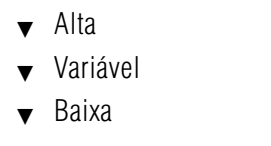 \\
\hline & $\begin{array}{l}\mathrm{Na} \text { avaliação dos resultados } \\
\text { do projeto }\end{array}$ & 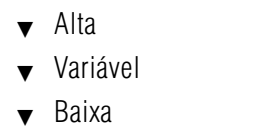 \\
\hline
\end{tabular}

Rap Rio de Janeiro 40(5):763-87, Set./Out. 2006 


\begin{tabular}{|c|c|c|}
\hline \multicolumn{2}{|r|}{ Quadro 5} & Efetividade: dimensões e indicadores \\
\hline Dimensões & Indicadores & Valores/Categorias \\
\hline \multirow[t]{3}{*}{ Eficácia } & Grau de atingimento de objetivos & $\begin{array}{ll}\boldsymbol{\nabla} & \text { Alto } \\
\boldsymbol{\nabla} & \text { Médio } \\
\boldsymbol{\nabla} & \text { Baixo }\end{array}$ \\
\hline & Grau de cumprimento de prazos & $\begin{array}{ll}\boldsymbol{\nabla} & \text { Alto } \\
\boldsymbol{\nabla} & \text { Médio } \\
\boldsymbol{\nabla} & \text { Baixo }\end{array}$ \\
\hline & Nível de qualidade dos produtos & $\begin{array}{l}\boldsymbol{\nabla} \text { Acima do esperado } \\
\boldsymbol{\nabla} \text { Conforme esperado } \\
\nabla \text { Abaixo do esperado }\end{array}$ \\
\hline \multirow[t]{3}{*}{ Eficiência } & $\begin{array}{l}\text { Resultados alcançados } \\
\text { (para a população-alvo) }\end{array}$ & \\
\hline & $\begin{array}{l}\text { Resultados alcançados } \\
\text { (para os demais stakeholders) }\end{array}$ & \\
\hline & $\begin{array}{l}\text { Percepção sobre a relação entre } \\
\text { os resultados do projeto e os recursos } \\
\text { utilizados }\end{array}$ & \\
\hline
\end{tabular}

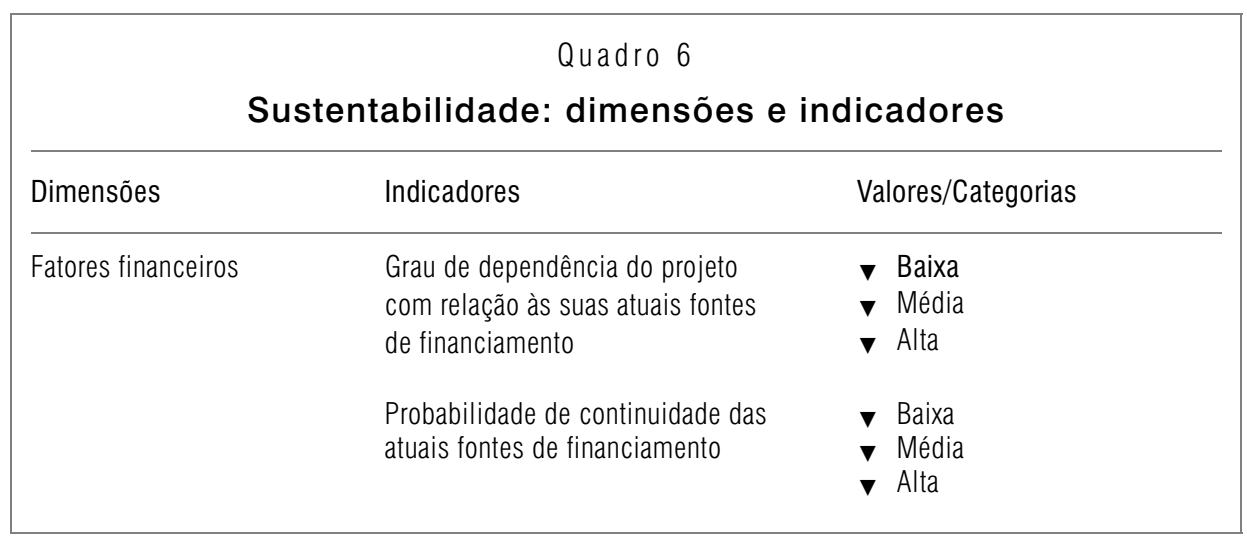

continua

Rap Rio de Janeiro 40(5):763-87, Set./Out. 2006 


\begin{tabular}{|c|c|c|}
\hline Dimensões & Indicadores & Valores/Categorias \\
\hline & $\begin{array}{l}\text { Capacidade do projeto de gerar receitas } \\
\text { para sua manutenção }\end{array}$ & $\begin{array}{ll}\boldsymbol{\nabla} & \text { Baixa } \\
\boldsymbol{\nabla} & \text { Média } \\
\boldsymbol{\nabla} & \text { Alta }\end{array}$ \\
\hline & $\begin{array}{l}\text { Capacidade de captação de recursos de } \\
\text { outras fontes para o projeto }\end{array}$ & $\begin{array}{ll}\boldsymbol{\nabla} & \text { Baixa } \\
\boldsymbol{\nabla} & \text { Média } \\
\boldsymbol{\nabla} & \text { Alta }\end{array}$ \\
\hline \multirow[t]{2}{*}{ Fatores sociais } & $\begin{array}{l}\text { Nível de participação dos beneficiários, } \\
\text { determinado pela dimensão participação, } \\
\text { do construto modelo de gestão }\end{array}$ & $\begin{array}{ll}\boldsymbol{\nabla} & \text { Alto } \\
\boldsymbol{\nabla} & \text { Médio } \\
\boldsymbol{\nabla} & \text { Baixo }\end{array}$ \\
\hline & $\begin{array}{l}\text { Classificação da população-alvo } \\
\text { com relação ao nível de } \\
\text { apropriação do projeto }\end{array}$ & $\begin{array}{ll}\boldsymbol{\nabla} & \text { Beneficiários } \\
\boldsymbol{\nabla} & \text { Clientes } \\
\boldsymbol{\nabla} & \text { Donos/gestores }\end{array}$ \\
\hline \multirow[t]{3}{*}{$\begin{array}{l}\text { Fatores institucionais e de } \\
\text { governabilidade }\end{array}$} & $\begin{array}{l}\text { Probabilidade de continuidade das } \\
\text { parcerias para desenvolvimento e } \\
\text { implementação do projeto }\end{array}$ & $\begin{array}{ll}\boldsymbol{\nabla} & \text { Alta } \\
\boldsymbol{\nabla} & \text { Média } \\
\boldsymbol{\nabla} & \text { Baixa }\end{array}$ \\
\hline & $\begin{array}{l}\text { Grau de apropriação do projeto } \\
\text { por parte do poder público }\end{array}$ & $\begin{array}{ll}\boldsymbol{\nabla} & \text { Alto } \\
\boldsymbol{\nabla} & \text { Médio } \\
\boldsymbol{\nabla} & \text { Baixo }\end{array}$ \\
\hline & $\begin{array}{l}\text { Vulnerabilidade do projeto a mudanças } \\
\text { políticas }\end{array}$ & $\begin{array}{ll}\boldsymbol{\nabla} & \text { Alta } \\
\boldsymbol{\nabla} & \text { Média } \\
\boldsymbol{\nabla} & \text { Baixa }\end{array}$ \\
\hline
\end{tabular}

\begin{tabular}{|c|c|c|}
\hline \multicolumn{3}{|c|}{$\begin{array}{c}\text { Quadro } 7 \\
\text { Satisfação dos stakeholders: indicadores }\end{array}$} \\
\hline Dimensão & Indicadores & Valores/Categorias \\
\hline \multirow[t]{2}{*}{$\begin{array}{l}\text { Satisfação dos públicos } \\
\text { afetados com os resultados } \\
\text { do projeto }\end{array}$} & Satisfação da população-alvo & $\begin{array}{ll}\boldsymbol{\nabla} & \text { Baixa } \\
\boldsymbol{\nabla} & \text { Média } \\
\boldsymbol{\nabla} & \text { Alta }\end{array}$ \\
\hline & Satisfação da comunidade local & $\begin{array}{ll}\boldsymbol{\nabla} & \text { Baixa } \\
\boldsymbol{\nabla} & \text { Média } \\
\boldsymbol{\nabla} & \text { Alta }\end{array}$ \\
\hline
\end{tabular}

continua

Rap Rio de Janeiro 40(5):763-87, Set./Out. 2006 


\begin{tabular}{|c|c|c|}
\hline Dimensão & Indicadores & Valores/Categorias \\
\hline & $\begin{array}{l}\text { Satisfação da equipe } \\
\text { do projeto }\end{array}$ & $\begin{array}{ll}\boldsymbol{\nabla} & \text { Baixa } \\
\boldsymbol{\nabla} & \text { Média } \\
\boldsymbol{\nabla} & \text { Alta }\end{array}$ \\
\hline & $\begin{array}{l}\text { Satisfação dos empregados das } \\
\text { empresas envolvidas }\end{array}$ & $\begin{array}{ll}\boldsymbol{\nabla} & \text { Baixa } \\
\boldsymbol{\nabla} & \text { Média } \\
\boldsymbol{\nabla} & \text { Alta }\end{array}$ \\
\hline & $\begin{array}{l}\text { Satisfação das instituições } \\
\text { parceiras }\end{array}$ & $\begin{array}{ll}\boldsymbol{\nabla} & \text { Baixa } \\
\boldsymbol{\nabla} & \text { Média } \\
\boldsymbol{\nabla} & \text { Alta }\end{array}$ \\
\hline & Satisfação do poder público & $\begin{array}{ll}\boldsymbol{\nabla} & \text { Baixa } \\
\boldsymbol{\nabla} & \text { Média } \\
\boldsymbol{\nabla} & \text { Alta }\end{array}$ \\
\hline & $\begin{array}{l}\text { Satisfação de outros públicos } \\
\text { considerados relevantes }\end{array}$ & $\begin{array}{ll}\boldsymbol{\nabla} & \text { Baixa } \\
\boldsymbol{\nabla} & \text { Média } \\
\boldsymbol{\nabla} & \text { Alta }\end{array}$ \\
\hline
\end{tabular}

\begin{tabular}{|llllll|}
\hline \multicolumn{5}{|c|}{ Quadro 8} \\
Tipologia de projetos segundo as dimensões da pesquisa \\
e seus indicadores
\end{tabular}


Projetos Sociais de Empresas no Brasil

\begin{tabular}{|c|c|c|c|c|c|}
\hline \multirow{2}{*}{ Dimensões } & \multirow{2}{*}{ Indicadores } & \multicolumn{4}{|c|}{ Correspondência entre os valores/categorias e o tipo de projeto } \\
\hline & & Tipo 1 & Tipo 2 & Tipo 3 & Tipo 4 \\
\hline Impacto & $\begin{array}{l}\text { Nível de } \\
\text { impacto que } \\
\text { pretende } \\
\text { causar }\end{array}$ & Superficial & Profundo & Profundo & Superficial \\
\hline $\begin{array}{l}\text { Produtos } \\
\text { resultantes }\end{array}$ & $\begin{array}{l}\text { Grau de } \\
\text { padronização } \\
\text { dos produtos }\end{array}$ & Padronizados & Padronizados & $\begin{array}{l}\text { Não- } \\
\text { padronizados }\end{array}$ & $\begin{array}{l}\text { Não- } \\
\text { padronizados }\end{array}$ \\
\hline \multirow[t]{2}{*}{$\begin{array}{l}\text { Atividades e } \\
\text { tarefas }\end{array}$} & $\begin{array}{l}\text { Grau de } \\
\text { rotinização }\end{array}$ & $\begin{array}{l}\text { Rotineiras e } \\
\text { repetitivas }\end{array}$ & $\begin{array}{l}\text { Rotineiras e } \\
\text { repetitivas }\end{array}$ & $\begin{array}{l}\text { Com constante } \\
\text { renovação }\end{array}$ & Pouco rotineiras \\
\hline & $\begin{array}{l}\text { Grau de } \\
\text { complexidade }\end{array}$ & Simples & Complexas & Complexas & $\begin{array}{l}\text { Relativamente } \\
\text { simples }\end{array}$ \\
\hline
\end{tabular}

\begin{tabular}{|c|c|c|c|c|c|}
\hline \multicolumn{6}{|c|}{$\begin{array}{c}\text { Tipologia de modelos de gestão segundo as dimensões } \\
\text { da pesquisa e seus indicadores }\end{array}$} \\
\hline \multirow[b]{2}{*}{ Dimensões } & \multirow[b]{2}{*}{ Indicadores } & \multicolumn{4}{|c|}{ Correspondência entre os valores/categorias e o tipo de projeto } \\
\hline & & Burocrático & Clientelar & Adhocrático & $\begin{array}{l}\text { Integração } \\
\text { homogênea }\end{array}$ \\
\hline $\begin{array}{l}\text { Orientação } \\
\text { da gestão }\end{array}$ & $\begin{array}{l}\text { Para oferta/para } \\
\text { demanda }\end{array}$ & $\begin{array}{l}\text { Gestão } \\
\text { orientada } \\
\text { para a oferta }\end{array}$ & $\begin{array}{l}\text { Gestão } \\
\text { orientada } \\
\text { para a } \\
\text { demanda }\end{array}$ & $\begin{array}{l}\text { Gestão } \\
\text { influenciada } \\
\text { pela demanda }\end{array}$ & $\begin{array}{l}\text { Gestão } \\
\text { influenciada } \\
\text { pela oferta }\end{array}$ \\
\hline \multirow[t]{2}{*}{$\begin{array}{l}\text { Controle e } \\
\text { medição de } \\
\text { desempenho }\end{array}$} & $\begin{array}{l}\text { Mecanismos } \\
\text { de controle }\end{array}$ & $\begin{array}{l}\text { Supervisão } \\
\text { direta e } \\
\text { hierárquica }\end{array}$ & $\begin{array}{l}\text { Padrões de } \\
\text { serviço e } \\
\text { satisfação do } \\
\text { beneficiário }\end{array}$ & $\begin{array}{l}\text { Auto-supervisão } \\
\text { e autocontrole } \\
\text { de grupos de } \\
\text { trabalho }\end{array}$ & $\begin{array}{l}\text { Sistemas de } \\
\text { controle e } \\
\text { gestão }\end{array}$ \\
\hline & $\begin{array}{l}\text { Critério básico } \\
\text { para medição } \\
\text { de desempenho }\end{array}$ & $\begin{array}{l}\text { Eficácia e } \\
\text { eficiência }\end{array}$ & $\begin{array}{l}\text { Satisfação } \\
\text { dos } \\
\text { stakeholders }\end{array}$ & Aprendizagem & $\begin{array}{l}\text { Coordenação } \\
\text { e gestão } \\
\text { de redes }\end{array}$ \\
\hline $\begin{array}{l}\text { Organização } \\
\text { do trabalho }\end{array}$ & $\begin{array}{l}\text { Estímulo ao } \\
\text { trabalho em } \\
\text { equipe }\end{array}$ & Baixo & Médio & Alto & Médio \\
\hline
\end{tabular}

continua

Rap Rio de Janeiro 40(5):763-87, Set./Out. 2006 


\begin{tabular}{|c|c|c|c|c|c|}
\hline \multirow[b]{2}{*}{ Dimensões } & \multirow[b]{2}{*}{ Indicadores } & \multicolumn{4}{|c|}{ Correspondência entre os valores/categorias e o tipo de projeto } \\
\hline & & Burocrático & Clientelar & Adhocrático & $\begin{array}{l}\text { Integração } \\
\text { homogênea }\end{array}$ \\
\hline & $\begin{array}{l}\text { Métodos de } \\
\text { trabalho }\end{array}$ & Programáveis & Mistos & $\begin{array}{l}\text { Não- } \\
\text { programáveis }\end{array}$ & Mistos \\
\hline Estrutura & Departamentalização & Funcional & $\begin{array}{l}\text { Por produto/ } \\
\text { por cliente }\end{array}$ & $\begin{array}{l}\text { Equipes de } \\
\text { trabalho, } \\
\text { matricial }\end{array}$ & $\begin{array}{l}\text { Divisões } \\
\text { territoriais e/ou } \\
\text { funcionais }\end{array}$ \\
\hline \multirow[t]{3}{*}{$\begin{array}{l}\text { Equipe do } \\
\text { projeto }\end{array}$} & $\begin{array}{l}\text { Conhecimento } \\
\text { necessário }\end{array}$ & Especializado & Intermediário & Generalizado & Intermediário \\
\hline & $\begin{array}{l}\text { Comprometimento } \\
\text { das pessoas } \\
\text { com os objetivos }\end{array}$ & Baixo & Moderado & Alto & Moderado \\
\hline & $\begin{array}{l}\text { Interesse da gestão } \\
\text { pela capacitação } \\
\text { das pessoas }\end{array}$ & Baixo & Alto & Alto & Moderado \\
\hline \multirow{2}{*}{$\begin{array}{l}\text { Flexibilidade } \\
\text { e percepção } \\
\text { do contexto }\end{array}$} & $\begin{array}{l}\text { Propensão a } \\
\text { mudanças }\end{array}$ & Baixa & Intermediária & Alta & Intermediária \\
\hline & Contexto & $\begin{array}{l}\text { Estável e } \\
\text { previsível }\end{array}$ & $\begin{array}{l}\text { Estável, } \\
\text { mas com } \\
\text { aleatoriedade }\end{array}$ & $\begin{array}{l}\text { Turbulento, } \\
\text { incerto }\end{array}$ & $\begin{array}{l}\text { Estável, } \\
\text { mas com } \\
\text { aleatoriedade }\end{array}$ \\
\hline \multirow[t]{3}{*}{ Liderança } & Estilo & Autocrática & Mista & Participativa & Mista \\
\hline & $\begin{array}{l}\text { Processo de } \\
\text { decisão }\end{array}$ & Centralizado & Variável & Descentralizado & Variável \\
\hline & $\begin{array}{l}\text { Canal para } \\
\text { resolução de } \\
\text { conflitos }\end{array}$ & Hierarquia & Comitê & $\begin{array}{l}\text { Equipes de } \\
\text { trabalho }\end{array}$ & Comitê \\
\hline \multirow[t]{2}{*}{ Participação } & Da população-alvo & Baixa & Variável & Alta & Variável \\
\hline & $\begin{array}{l}\text { Da equipe } \\
\text { operacional } \\
\text { do projeto }\end{array}$ & Baixa & Variável & Alta & Variável \\
\hline
\end{tabular}

\section{Aplicações do arcabouço conceitual}

Uma das aplicações do arcabouço é servir de lista de referência para a confecção de instrumentos de coleta de dados e análise de resultados de pesquisas empíricas sobre projetos sociais, das seguintes formas:

Rap Rio de Janeiro 40(5):763-87, Set./Out. 2006 
$\checkmark$ questionários estruturados - recomendamos que sejam formuladas questões para cada um dos indicadores que compõem as dimensões constitutivas dos construtos;

v roteiros para entrevistas em profundidade - para uma compreensão mais profunda das questões pertinentes, que não podem ser captadas apenas por meio de questionário. Assim, podem ser mais bem exploradas as relações entre os fatores contemplados pelo arcabouço (representadas pelas setas na figura) e suas implicações para a efetividade, sustentabilidade e satisfação dos stakeholders;

v protocolos para estudos de caso - o arcabouço poderá, adicionalmente, orientar o desenvolvimento de protocolos para estudos de caso simples ou múltiplos, orientando o tipo de dados que deve ser coletado de diferentes fontes de evidência, a fim de se obter uma triangulação de informações, para uma maior confiabilidade dos resultados.

O arcabouço também poderá ser utilizado como ferramenta gerencial por gestores de projetos sociais das empresas e instituições parceiras. O detalhamento dos construtos, dimensões e indicadores oferece a possibilidade de analisar a adequação entre o modelo de gestão adotado e as características do projeto, bem como as implicações dos diversos outros fatores e de suas interrelações para as medidas de desempenho do projeto - efetividade, sustentabilidade e satisfação dos stakeholders. Esse tipo de análise permite a identificação de hiatos, inconsistências e pontos de melhoria a serem trabalhados.

Vale ressaltar que a análise dos construtos configuração institucional, tipo de projeto e modelo de gestão deve ser feita de forma relacional, ou seja, analisando-se sua combinação, adequação e congruência. Para o construto efetividade, é desejável que as dimensões assumam os valores mais favoráveis, assinalados em negrito no quadro 5 . A análise da dimensão eficiência deve ser feita relativamente aos objetivos definidos para o projeto. Para satisfação dos stakeholders, quanto mais altos os valores, melhor.

O construto sustentabilidade deve ser analisado com cautela. De forma geral, é desejável que suas dimensões assumam os valores assinalados em negrito no quadro 6. No entanto, a sustentabilidade pode perder sua importância em projetos pontuais, que não tenham como objetivo causar impactos profundos.

Percebemos que a participação dos beneficiários é, ao mesmo tempo, uma dimensão do modelo de gestão e componente da dimensão social da sustentabilidade. Deve ser feita uma análise cuidadosa do grau de participação com relação ao tipo de projeto e às mudanças que se pretende alcançar com o mesmo. Quanto mais profundo o impacto e maiores as mudanças pretendidas, maior a necessidade de participação da população. Assim, pode-se questionar e analisar os verdadeiros objetivos do projeto. 


\section{Considerações finais}

Este artigo teve como objetivo apresentar o arcabouço conceitual desenvolvido como parte de uma pesquisa com vistas à investigação dos modelos de gestão adotados por empresas, no Brasil na condução de seus projetos sociais.

$\mathrm{O}$ arcabouço conceitual apresentado não tem a pretensão de ser exaustivo, mas de contribuir tanto para a área de pesquisa de RSC, mostrando um caminho possível de ser trilhado nas pesquisas de campo sobre investimentos sociais de empresas, quanto para sua prática no dia-a-dia das empresas, ajudando na análise da adequação entre os diversos fatores intervenientes e de suas implicações para o desempenho do projeto.

Esperamos que as pesquisas desenvolvidas com o auxílio deste artigo possam enriquecer o próprio arcabouço, por meio da detecção de outros possíveis construtos a serem investigados, da discussão e validação das dimensões propostas, da proposição de novas dimensões e indicadores e de uma melhor compreensão das relações entre seus elementos constitutivos.

\section{Referências bibliográficas}

BID/ILPES. Gestión y control de proyectos - Programa de Capacitación BID/Ilpes. agosto 2000. Disponível em: <www.eclac.org/publicaciones>. Acesso em: 20 nov. 2004.

CEATS-USP. Estratégias de empresas no Brasil: atuação social e voluntariado. São Paulo: Programa Voluntários, 1999.

- Alianças estratégicas intersetoriais para atuação social. Disponível em: <www.fia. com.br/CEATS/publica.php>. Acesso em: 18 mar. 2005.

CEPAL (COMISSÃO ECONÔMICA PARA AMÉRICA LATINA E CARIBE). Manual de formulação e avaliação de projetos sociais. Cepal, 1995.

. Gestión de programas sociales em América Latina. Série Políticas Sociales, Santiago de Chile: Cepal, v. 1, n. 25, 1998.

COHEN, Ernesto; FRANCO, Rolando. Avaliação de projetos sociais. Petrópolis: Vozes, 1998.

COSTA, Frederico L.; CASTANHAR, José Cezar. Avaliação de programas públicos: desafios conceituais e metodológicos. Revista de Administração Pública, v. 37, n. 5, set./out. 2003.

FISCHER, Rosa M. et al. Monitoramento de projetos sociais: um desafio para as alianças intersetoriais. In: ENANPAD 2003. Anais... Atibaia, set. 2003. CD-ROM.

Rap Rio de Janeiro 40(5):763-87, Set./Out. 2006 
GIFE. Censo retrata parcela do investimento social privado no Brasil. Disponível em: $<$ www.gife.org.br>. Acesso em: 10 jun. 2001.

IPEA. Bondade ou interesse? Como e porque as empresas atuam no social. Set. 2001.

A iniciativa privada e o espírito público: a ação social das empresas privadas no Brasil. Informações à imprensa. Jun. 2002.

ISMERIO, Marcia Gomes. Desenvolvimento sustentável: o social como desafio na contemporaneidade. 1999. Dissertação (Mestrado) — Universidade Federal do Rio de Janeiro, Instituto de Psicologia, Programa Eicos, Cátedra Unesco de Desenvolvimento Durável, Rio de Janeiro, 1999.

KERLINGER, Fred N. Foundations of behavioral research. Holt, Reinhart and Winston, 1973.

MOKATE, Karen. Eficácia, eficiencia, equidad y sostenibilidad: qué queremos decir? Banco Interamericano de Desarrollo, Instituto Interamericano para el Desarrollo Social (Indes), 2002.

NARAYAN, Deepa. Designing community based development. Social Development Papers, The World Bank, n. 7, June 1995.

NOGUEIRA, Roberto Martínez. Los proyectos sociales: de la certeza omnipotente al comportamiento estratégico. Santiago de Chile: Cepal, 1998.

SACHS, Ignacy. Social sustainability and whole development: exploring the dimensions of sustainable development. In: BECKER, Egon; JAHN, Thomas (Orgs.). Sustainability and social sciences: a cross-disciplinary approach to integrating environmental considerations into theoretical reorientation. Zed Books, 2000.

TANKERSLEY, Ady Marina. Meio ambiente e ação empresarial numa abordagem integradora. 1994. Dissertação (Mestrado) - Universidade Federal do Rio de Janeiro, Instituto de Psicologia, Programa Eicos, Cátedra Unesco de Desenvolvimento Durável. Rio de Janeiro, 1994.

THOMPSON, Andres A.; TANCREDI, Francisco B.; KISIL, Marcos. New partnerships for social development: business and the third sector. International Journal of Public Administration, p. 1359-1380, May/Aug., 2000. 